\title{
COARSENING IN ONE DIMENSION: INVARIANT AND ASYMPTOTIC STATES
}

\author{
EMANUEL A. LAZAR AND ROBIN PEMANTLE
}

\begin{abstract}
We study a coarsening process of one-dimensional cell complexes. We show that if cell boundaries move with velocities proportional to the difference in size of neighboring cells, then the average cell size grows at a prescribed exponential rate and the Poisson distribution is precisely invariant for the distribution of the whole process, rescaled in space by its average growth rate. We present numerical evidence toward the following universality conjecture: starting from any finite mean stationary renewal process, the system when rescaled by $e^{-2 t}$ converges to a Poisson point process. For a limited case, this makes precise what has been observed previously in experiments and simulations, and lays the foundation for a theory of universal asymptotic states of dynamical cell complexes.
\end{abstract}

Key words and phrases: Poisson, divergence, evolution.

Subject classification Primary: 60A10; Secondary: 53C44, 82C21.

\section{INTRODUCTION}

Many physical systems can be abstracted as cell complexes whose geometry and topology change over time through deterministic evolution equations. Soap foams and polycrystalline metals are two examples of such systems that evolve under a generalized curvature flow to reduce an energy associated with the co-dimension 1 cells. Over time, some cells grow while others shrink and disappear. This is sometimes called a coarsening process, because as cells disappear, the average size of the remaining cells monotonically increases [Laz11].

A remarkable self-similarity property has long been observed in both experiments and numerical simulations of these systems: if an arbitrary cell complex is allowed to evolve by fixed evolution equations, then its scale-independent properties will converge, in a statistical sense, to a time-independent set of properties. For example, the distribution of normalized cell sizes converges to some fixed distribution, even as the system itself continues to evolve [Laz11, LMMS11, MLMS12. This general behavior is sometimes referred to as statistical self-similarity [Mul86, MV89]. A second remarkable universality property is also observed: these self-similar properties are largely independent of the initial conditions, and instead depend on the deterministic evolution dynamics.

The primary aim of this paper is to give a rigorous analysis of a one-dimensional coarsening process in which a limit theorem can be proved. The second goal is to explore the degree to which 


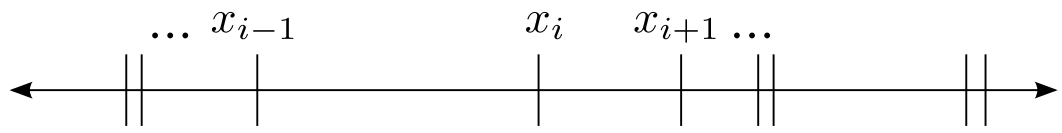

Figure 1. A one-dimensional cell complex with a few of the cell boundaries $x_{i}$ labeled.

the limiting behavior is independent of initial conditions. These will be carried out in the context of a particular one-dimensional system which can be viewed as one-dimensional curvature flow.

We parametrize configurations by the locations $x_{i}$ of the boundaries between the 1-cells. The particular system we initially consider is one in which cell boundaries move with a velocity proportional to the difference of the sizes of two neighboring 1-cells. We let $X=\left(x_{i}\right) \in \mathbb{R}$ be an ordered set of points on a one-dimensional manifold, as illustrated in Figure 1, The evolution of this system is governed by two rules:

$$
\begin{gathered}
\qquad \frac{d x_{i}}{d t}=-x_{i-1}+2 x_{i}-x_{x+1} \\
\text { If } x_{i}=x_{j} \text { for } i \neq j: \quad \text { we remove } x_{j} \text { and reindex the remaining points. }
\end{gathered}
$$

Rule 2 prevents points from crossing one another. It is straightforward to see that 1-cells that are larger than their two neighbors grow, while those smaller than their neighbors shrink.

Previous Work. Important work has been done in studying one-dimensional dynamical cell complexes, though these systems are often known by the names clustering or coagulation systems. Carr and Pego [CP92] considered a model in which at discrete time steps the shortest interval in a finite system is taken and joined with its neighbors. They use Laplace transforms to establish the existence of a self-similar solution. This system makes use of discrete time steps and "long-range" interaction. Derrida Der95, Der97] considered one-dimensional Ising and Potts models at zero temperature and considered the rates at which these systems coarsen. He showed that if the initial condition is random, the system enters a scaling regime where the average size of domains grows as $t^{1 / 2}$. Nontrivial exponents that govern other properties of these coarsening systems are also calculated. Hunderi and Ryum [HR96, HRW79] considered a system in which $\frac{d x_{i}}{d t}=\frac{1}{x_{i+1}-x_{i}}-\frac{1}{x_{i}-x_{i-1}}$. They used numerical simulations to demonstrate that a size correlation develops between neighboring cells; this correlation calls into question the reliability of mean field theories [FRS92, which tend to ignore size correlations between neighboring cells, in analyzing these systems.

Mullins Mul91 considered a slightly more general version of the system considered in this paper, and established some very preliminary results for these systems. Unfortunately, despite work of Mullins and of others, Mullins' conclusion, "Very little is known, however, about the general conditions under which self-similarity is to be expected" remains true nearly twenty-five years later. 


\section{Results}

Our main result is the invariance of the Poisson distribution for cell boundaries under the evolution (1.1)-(1.2). We begin with an analysis of what happens with a finite number of such points, moving in a finite interval. For boundary conditions, here and throughout, we impose two extra particles that remain stationary at the endpoints 0 and 1.

Theorem 2.1. Begin with $n$ points chosen uniformly on $[0,1]$. The law after time $t$ is that of $Z$ points chosen uniformly and independently on $[0,1]$ where $Z$ is a binomial with parameters $n$ and $e^{-2 t}$.

Because these points are moving around, colliding with each other and coalescing, we refer to them henceforth as particles. Some consequences follow immediately from Theorem 2.1, properties of the binomial and Poisson distributions, and scaling invariance. Let $\mathcal{P}(\lambda, L)$ denote the law of a Poisson process of intensity $\lambda$ times Lebesgue measure on $[-L, L]$; alternatively it can be described as the law of $Z$ IID uniform points in $[-L, L]$ when $Z$ has Poisson distribution with mean $\lambda$.

\section{Corollary 2.2.}

1. Starting from $n$ particles uniformly distributed in $[-L, L]$, the number of particles in a given subset $A \subseteq[-L, L]$ after time $t$ has binomial distribution

$$
\operatorname{Bin}\left(n, e^{-2 t} \frac{|A|}{2 L}\right) \text {. }
$$

2. Starting from $\mathcal{P}(\lambda, L)$, the law of the configuration after time $t$ is a Poisson configuration with intensity $\lambda e^{-2 t}$ times Lebesgue measure on $[-L, L]$.

3. For $M \geq L>0$, let $\mathcal{L}(M, L)$ be the law of the time $t$ configuration restricted to $[-L, L]$, starting from $\mathcal{P}(\lambda, M)$. Then the law $\mathcal{L}(M, L)$ does not depend on $M$.

Next we turn to the infinite process. The first thing is to establish an existence result. This is not trivial because of the possibility of influence coming in from infinity in finite time and because at collision times, which are dense, the trajectory of every particle become non-analytic. In Section 7 we will define the space $\Omega$ of infinite particle configurations and prove the following result.

Theorem 2.3 (existence of a weak solution for the infinite system). Let $\Omega$ be the space of continuous trajectories of infinite configurations defined in Section 7 . For every $\lambda>0$ there exists a weak solution to (1.1) with initial condition Poisson of intensity $\lambda$, in the sense of Definition 7.2. Any such solution has the following properties.

(i) The trajectory of each particle is always differentiable from the right.

(ii) Excluding a measure zero set of initial configurations, the collision times will be distinct.

(iii) At all times that a particle is not involved in a collision, that particle's position has a two-sided derivative. 
The infinite model allows for a statement of Poisson invariance cleaner than the one in Corollary 2.2 ,

Theorem 2.4. Under any weak solution $\mathbb{P}_{\lambda}$, the time $t$ law of the configuration is Poisson with intensity $\lambda e^{-2 t}$.

\section{Evolution ON A Finite INTERVAL}

In order to define the process on a finite interval, we need to choose how the first and last particles evolve. As previously mentioned, rather than using periodic boundary conditions, we impose extra particles at the endpoints that never move.

The space of configurations of $n$ particles on $[0,1]$ is the simplex

$$
\Delta_{n}:=\left\{\mathbf{x}=\left(0, x_{1}, \ldots, x_{n}, 1\right): 0=x_{0} \leq x_{1} \leq \cdots \leq x_{n} \leq x_{n+1}=1\right\}
$$

We include the degenerate simplex $\Delta_{0}:=\{(0,1)\}$. The interior $\Delta_{n}^{o}$ of $\Delta_{n}$ is the set of configurations with all particles distinct from each other and from the endpoints $\{0,1\}$. After a collision, two particles, say $x_{i}$ and $x_{i+1}$, get stuck together. The configuration is no longer in $\Delta_{n}^{o}$, rather it is now on the boundary $\partial \Delta_{n}$. The boundary is composed of faces of various dimensions defined below as images of open simplices under various embeddings. On each of these, the evolution satisfies a different rule. Rather than think of this as a single discontinuous rule on the closed simplex, we think of the particle as entering the $(n-1)$-simplex at the first collision time.

To make this precise, we define a set of embeddings as follows. For integers $n \geq 0$ and $k \in[0, n+1]$, define the map $\iota_{n, k}: \Delta_{n} \rightarrow \Delta_{n+1}$ by the formula

$$
\iota_{n, k}\left(0, x_{1}, \ldots, x_{n}, 1\right)= \begin{cases}\left(0,0, x_{1}, \ldots, x_{n}, 1\right) & k=0 \\ \left(0, x_{1}, \ldots, x_{n}, 1,1\right) & k=n+1 \\ \left(0, x_{1}, \ldots, x_{k}, x_{k}, \ldots, x_{n}, 1\right) & 1 \leq k \leq n\end{cases}
$$

Define the projection map from $\Delta_{n}$ to the union of $\Delta_{k}^{o}$ for $k \leq n$ by letting $\pi\left(0, x_{1}, \ldots x_{n}, 1\right)$ be the result of omitting repeated entries. Then $\pi \circ \iota_{n-1, k}(\mathbf{x})=\mathbf{x}$ for each $\mathbf{x} \in \Delta_{n-1}^{o}$ and each $k \in[0, n]$. More generally, any composition of maps $\iota_{n-1, k_{n-1}} \circ \cdots \circ \iota_{m, k_{m}}$ is inverted by $\pi$ on $\Delta_{m}^{o}$.

We now define an evolution on the configuration space $\Delta:=\biguplus_{n} \Delta_{n}^{o}$, the disjoint union of $n$-point configuration spaces on $[0,1]$. The evolution is defined as a set of time $t$ maps $\left\{\Phi_{t}\right\}$ on $\Delta$ and constructed by induction on the dimension $n$ of the stratum in $\Delta$. The base step of the induction is to define $\Phi_{t}(\mathbf{x}) \equiv 0$ on $\Delta_{0}$. 
Let $A_{n}$ denote the $(n+2) \times(n+2)$ matrix defined by

$$
A_{n}=\left[\begin{array}{cccccc}
0 & 0 & 0 & 0 & \cdots & 0 \\
-1 & 2 & -1 & 0 & \cdots & 0 \\
0 & -1 & 2 & -1 & \cdots & 0 \\
\vdots & \vdots & & & & \vdots \\
0 & \cdots & 0 & -1 & 2 & -1 \\
0 & \cdots & 0 & 0 & 0 & 0
\end{array}\right] .
$$

The differential equation $\mathbf{x}^{\prime}=A_{n} \mathbf{x}$ defines a flow on $\Delta_{n}^{o}$. For $\mathbf{x} \in \Delta_{n}^{o}$, let

$$
\tau(\mathbf{x}):=\inf \left\{t: e^{t A_{n}} \mathbf{x} \in \partial \Delta_{n}\right\}
$$

We may now recursively define

$$
\Phi_{t}(\mathbf{x}):= \begin{cases}e^{t A_{n}} \mathbf{x} & t<\tau(\mathbf{x}) \\ \Phi_{t-\tau(\mathbf{x})}\left(\pi\left(e^{\tau A_{n}} \mathbf{x}\right)\right) & t \geq \tau(\mathbf{x})\end{cases}
$$

Informally, flow by $\mathbf{x}^{\prime}=A_{n} \mathbf{x}$ until you reach the boundary, then collapse whichever points need to be collapsed and continue inductively ( $\mathrm{run}^{\prime}=A_{m} \mathbf{x}$ where $m$ is the new number of particles, and so on). For all but a set of measure zero of initial points, each collision will reduce the number of particles by precisely 1 ; however, the flow is well defined even for simultaneous coalescences, in which case $\pi$ drops dimension by more than 1 .

Remark. One can define the evolution directly on $\Delta_{n}$. At time $\tau(\mathbf{x})$, let $F$ be the unique face of $\Delta_{n}$ to which $\mathbf{x}$ is interior. Let $\iota$ be the composition of maps $\iota_{m, k}$ inverted by $\pi$ on $F$; the map $\iota$ is unique even though it may be represented by a sequence of compositions in many ways. Instead of mapping $\mathbf{x}$ to the projection $\pi\left(\Phi_{\tau(\mathbf{x})}(\mathbf{x})\right)$ of its time $\tau$ image, continue the evolution via

$$
\Phi_{\tau+s}(\mathbf{x}):=\iota\left(\Phi_{s}\left(\pi\left(e^{\tau A_{n}} \mathbf{x}\right)\right) .\right.
$$

Informally, the evolution on $\Delta_{n}$ keeps track not only of the coalesced system but of which original particles coalesced at what is now particle $i$.

\section{The time $t$ PRE-IMAge of A Finite CONFiguration}

The forward flow goes until it hits the boundary, jumps down a dimension, and continues. The reverse flow goes inward away from the boundary, but unlike the forward flow, it is capable at any time of reinterpreting its position in $\Delta_{m}$ as a position on $\partial \Delta_{m+1}$, in which case it jumps up a dimension and begins to flow inward from the boundary of the bigger simplex. Not only that, but it can choose to jump up in any of $m+2$ ways: any of the particles can split into two, or a particle can enter at 0 or 1 . In this section we characterize all possible reverse trajectories. First we check that the flow, when not splitting, does indeed flow inward. 
Lemma 4.1. For $t>0$, the map $\mathbf{x} \mapsto e^{-t A_{n}} \mathbf{x}$ is one to one on $\mathbb{R}^{n+2}$ and maps $\triangle_{n}$ into its own interior.

Proof: Letting $y_{j}=x_{j+1}-x_{j}$, the ODE $\mathbf{x}^{\prime}=-A_{n} \mathbf{x}$ induces an ODE $\mathbf{y}^{\prime}=B_{n} \mathbf{y}$ where $B_{n}$ is the $(n+1) \times(n+1)$ matrix with ones on the first super- and sub-diagonal, and -2 on the main diagonal except that $B_{1,1}=B_{n+1, n+1}=-1$, not -2 . Each $\left(B_{n} \mathbf{y}\right)_{j}$ is a linear combination of coordinates of $\mathbf{y}$ with the only negative contribution coming from $y_{j}$; hence if $\mathbf{y} \in\left(\mathbb{R}^{+}\right)^{n}$ and $y_{j}=0$ then $\left(B_{n} \mathbf{y}\right)_{j} \geq 0$. By Nagumo's Theorem [Aub77] (or see [Har72, Theorem 1]), solutions to $\mathbf{y}^{\prime}=B_{n} \mathbf{y}$ never leave the nonnegative orthant. It follows that the coordinates $x_{j}$ never collide.

Now fix integers $n \geq m \geq 0$ and a point $\mathbf{y} \in \Delta_{m}^{o}$, and define the following notation for the time $t$ dimension $n$ pre-image:

$$
\Phi_{t}^{-1}(\mathbf{y} ; n):=\left\{\mathbf{x} \in \Delta_{n}: \mathbf{y}=\Phi_{t}(\mathbf{x})\right\} .
$$

We show that $\Phi_{t}^{-1}(\mathbf{y} ; n)$ has a one to one parametrization by the sequence of times at which the split occurs and the choice of which particle splits at each time.

To define this formally, let $T(t ; k)$ be the $k$-simplex of vectors $\mathbf{t}$ satisfying $0 \leq t_{1} \leq \cdots \leq t_{k} \leq t$. Define

$$
S(n ; m):=\left\{\left(k_{1}, \ldots, k_{n-m}\right): 0 \leq k_{i} \leq n-i+1\right\} .
$$

As time passes backward through $t_{k}$, one of the existing $n-k$ particles splits; these choices are what is encoded by an element of $S(n ; m)$.

Proposition 4.2. Fix integers $m \leq n$ and a positive real $t$. Let $k:=n-m$. There is a map $\eta$ from $\Delta_{m} \times T(t ; k) \times S(n ; k)$ to $\Delta_{n}$ such that if $\mathbf{x} \in \eta(\mathbf{y}, \mathbf{t}, \mathbf{s})$ then

(i) $\Phi_{t}(\mathbf{x})=\mathbf{y}$

(ii) For each $1 \leq j \leq k$, the first entry time $\tau_{j}$ of $\Phi$.(x) into $\Delta_{n-j}$ is equal to $t_{j}$

(iii) For each $1 \leq j \leq k$ the coalescence at time $\tau_{j}$ occurs between coordinates $s_{j}$ and $s_{j-1}$. If $\tau_{j}=\cdots=\tau_{j+r}$, this is taken to mean that the $r+1$ coalescences at this time are those identifying $s_{j+i}$ with $s_{j+i-1}$ for $i=0, \ldots, r$.

Proof: We construct the map $\eta$ explicitly. Run the clock backwards starting at time $t$, evolving backwards via $e^{-t A_{m}}$. By Lemma 4.1, the point stays in $\Delta_{m}^{o}$. When the clock reaches $t_{k}$, jump from $\Delta_{m}$ to $\Delta_{m+1}$ via the embedding $\iota_{m, s_{k}}$. Continue evolving backwards via $e^{-t A_{m+1}}$ until the clock reaches time $t_{k-1}$, then jump up to $\Delta_{m+2}$ via $\iota_{m+1, s_{k-1}}$. Continue in this manner, reaching $\Delta_{n}$ at time $t_{1}$ and evolving in $\Delta_{n}$ by $e^{-t A_{n}}$ for a backward time $t_{1}$ until the clock says zero.

With this construction of $\eta$, the consequences of the proposition are easily checked by induction on $k$. When $k=0$ the vectors $\mathbf{t}$ and $\mathbf{s}$ are empty and the only fact to check is that $e^{-t A}$ inverts $e^{t A}$. For $k>1$, evolving back in time by $t-t_{k}$ yields a point $\mathbf{y}^{\prime}:=e^{-t A_{m}} \mathbf{y}$ and a point $\mathbf{y}^{\prime \prime}:=\iota_{m+1, s_{k}} \mathbf{y}^{\prime}$. Apply the induction hypothesis to $\mathbf{x}=\eta\left(\mathbf{y}^{\prime}, \mathbf{t}^{\prime}, \mathbf{s}^{\prime}\right)$ where $\mathbf{t}^{\prime}$ and $\mathbf{s}^{\prime}$ drop that last coordinates of 
$\mathbf{t}$ and $\mathbf{s}$ respectively to see that $\Phi_{t_{k}-}(\mathbf{x}):=\lim _{s \uparrow t_{k}} \Phi_{s}(\mathbf{x})=\mathbf{y}^{\prime \prime}$. Thus, $\Phi_{t}(\mathbf{x})=\Phi_{t-t_{k}}\left(\Phi_{t_{k}}(\mathbf{x})\right)=$ $\Phi_{t-t_{k}}\left(\mathbf{y}^{\prime}\right)=\mathbf{y}$. One final coalescence occurs at time $t_{k}$ in coordinates $s_{k}$ and $s_{k}-1$. This completes the induction.

\section{INVARIANCE THEOREM FOR FINITE CONFIGURATIONS}

This section is devoted to the proof of Theorem 2.1 and its consequences. We reduce it to Lemma 5.2 below, prove the further Lemma 5.3 , then prove Lemma 5.2. It will be helpful to define a certain continuous time Markov chain on the nonnegative integers. It is a pure death chain, that is, transitions from $n$ are allowed only to $n-1$. The rate at which $n$ transitions to $n-1$ is $2 n$. If $\left\{X_{t}: t \geq 0\right\}$ is such a Markov chain, one interpretation is that $X_{t}$ is the number of particles alive at time $t$, where each particle independently dies at rate 2 (meaning, after an exponential random time with mean 1/2). Denote the transition probabilities for this chain by $p_{t}$. Thus, $p_{t}(n, m)$ is the probability, starting with $n$ particles, that precisely $m$ are alive at time $t$. Saying it another way,

$$
p_{t}(n, \cdot) \sim \operatorname{Bin}\left(n, e^{-2 t}\right) .
$$

Let $g\left(t_{1}, \ldots, t_{k}\right)=g\left(n ; t_{1}, \ldots, t_{k}\right)$ denote the density for the first $k$ transitions starting from $n$. An explicit formula for $g$ is

$$
g\left(n ; t_{1}, \ldots, t_{k}\right)=2^{k} n(n-1) \cdots(n-k+1) \exp \left(-\sum_{j=1}^{k} 2(n-j+1)\left(t_{j}-t_{j-1}\right)\right)
$$

where $t_{0}=0$ by convention.

Normalized Lebesgue measure on $\Delta_{n}$ is the probability measure $\mu_{n}$ whose density with respect to Lebesgue measure is $n !$. We first prove a special case of Theorem 2.1.

Lemma 5.1. Let $B$ be any Borel subset of $\triangle_{n}$ and let $t$ be any positive real number. Then

$$
\mu_{n}\left\{x \in \triangle_{n}: \tau_{\mathbf{x}}>t \text { and } e^{t A} \mathbf{x} \in B\right\}=e^{-2 n t} \mu_{n}(B) .
$$

Consequently, after time $t$ the probability of still having $n$ points is $e^{-2 n t}$, and conditional on this the points are independently and uniformly distributed.

Proof: The divergence of the vector field $F(\mathbf{x})=A_{n} \mathbf{x}$ on $\mathbb{R}^{n+2}$ is equal to $2 n$. It follows that the Jacobian of the map $e^{t A}$ on $\mathbb{R}^{n}$ is equal to $e^{2 n t}$; this is easily deduced from the fact that a divergence free flow has Jacobian equal to one [Lee03, Proposition 18.18] applied to the map $e^{-2 t} e^{t A}$. Also, the time $t$ map on $\mathbb{R}^{n}$ is one to one and is inverted by $e^{-t A}$ [Lee03, Theorem 17.8]. It follows that the Lebesgue measure of $e^{-t A} B$ is equal to $e^{-2 n t}$ times the Lebesgue measure of $B$. By Lemma 4.1, the set $e^{-t A}[B]$ is contained in $\triangle_{n}$ and for each $\mathbf{x} \in e^{-t A}[B]$ the stopping time $\tau_{\mathbf{x}}$ is greater than $t$ (apply the lemma for all $s \leq t$ ). The conclusion of the lemma follows.

To see that that yields the special case of Theorem 2.1 let $B$ be a subset of the interior of $\Delta_{n}$. Then the probability, starting with a uniform random point of $\Delta_{n}$, of being in $B$ at time $t$ is the $\mu_{n}$ 
measure of points $\mathbf{x} \in \Delta_{n}$ that evolve in time $t$ to a point in $B$. By Lemma 5.1 this is $e^{-2 n t} \mu_{n}(B)$. As the probability of a $\operatorname{Bin}\left(n, e^{-2 t}\right)$ random variable being equal to $n$ is precisely $e^{-2 n t}$, the conclusion of Theorem 2.1 is verified in this case.

The full theorem is a consequence of the next result. Extend the notation for the map $\eta$ so that it acts on sets: for $B \subseteq \Delta_{m}, T \subseteq T(t ; k)$ and $S \subseteq S(n ; k)$ we define

$$
\eta[B, T, S]:=\{\eta(\mathbf{y}, \mathbf{t}, \mathbf{s}): \mathbf{y} \in B, \mathbf{t} \in T, \mathbf{s} \in S\} .
$$

In general, for $K \subseteq \Delta_{m} \times T(t ; k) \times S(n ; k)$ we denote $\eta[K]:=\{\eta(\mathbf{y}, \mathbf{t}, \mathbf{s}):(\mathbf{y}, \mathbf{t}, \mathbf{s}) \in K\}$.

Lemma 5.2. Let $m=n-k \leq n$, let $t>0$, and let $K=K_{0} \times S(n ; k)$ be the product of a measurable subset of $\Delta_{m} \times T(t ; k)$ with all of $S(n ; k)$. Let $g$ be the density defined in equation (5.2). Then

$$
\mu_{n}(\eta(K))=\int_{K} g(\mathbf{t}) d \mu_{L}(\mathbf{y}) d \mathbf{t} .
$$

This lemma says that Lebesgue measure on $\Delta_{n}$ pulled back by $\eta$ yields the product of normalized Lebesgue measure with the measure on $T(t ; k)$ having density $g$, provided that one sums over all possible embedding sequences $\mathbf{s}$. Before proving it, let us check that it implies Theorem 2.1 Setting $K=B \times T(t ; k)$ makes $\eta(K)$ the set of all $\mathbf{x}$ for which $\Phi_{t}(\mathbf{x}) \in B$. The integral in (5.3) factors as a product in the $\mathbf{y}$ and $\mathbf{t}$ variables, resulting in

$$
\mu_{n}(K)=\mu_{m}(B) p_{t}(n, m)
$$

The left-hand side is $\mathbb{P}\left(\Phi_{t}(\mathbf{x}) \in B\right)$ and the right-hand side is the normalized Lebesgue measure of $B$ times the probability of $\operatorname{Bin}\left(n, e^{-2 t}\right)=m$. This proves Theorem 2.1.

There are two tricky points in establishing (5.3). One is seeing how the product of $m$ dimensional measure on $\Delta_{m}$ and $k$ dimensional measure on $T(t ; k)$ maps to $m+k=n$ dimensional measure on $\Delta_{n}$. In fact the bifurcation at a variable time $s \in[t, t+d t]$ results in a product with a spatial interval proportional to the normal velocity of of the flow toward the boundary of $\Delta_{n}$. The second tricky point is why the total of these factors involving normal velocities is always constant if one sums over all sequences s. For the first of these two tricky points we record the following lemma, for which all notation is declared to be local.

Lemma 5.3. Let $H$ be a subset of $\mathbb{R}^{d}$ which is locally a halfspace with boundary $K$. Let $B$ be a Borel subset of $K$, let $F$ be a vector field on $H$ with $F \cdot \mathbf{n}>0$ on $K$ where $\mathbf{n}$ is the inward pointing unit normal. Let $\left\{\Phi_{t}\right\}$ denote the flow associated with the differential equation $\mathbf{x}^{\prime}=F(\mathbf{x})$. Let $B_{t} \subseteq H$ be the set $\left\{\Phi_{s}(\mathbf{x}): \mathbf{x} \in B, 0 \leq s \leq t\right\}$. Then

$$
\lim _{t \downarrow 0} \frac{\left|B_{t}\right|}{t}=\int_{B} F(\mathbf{x}) \cdot \mathbf{n}(\mathbf{x}) d \mathbf{x} .
$$


Proof: The map $\Phi: K \times[0, t]$ taking $(\mathbf{x}, s)$ to $\Phi_{s}(\mathbf{x})$ has Jacobian $J(\mathbf{x})=F(\mathbf{x}) \cdot \mathbf{n}(\mathbf{x})$ at the point $(\mathbf{x}, 0)$ because it has a matrix representation in blocks of dimension $d-1$ and 1 of

$$
\left[\begin{array}{c|c}
I_{d-1} & * \\
\hline 0 & F(\mathbf{x})
\end{array}\right]
$$

in local coordinates $K \times[0, \infty)$. The measure of $B_{t}$ is equal to $\int_{B \times[0, t]} J(\mathbf{x}, t)(d \mathbf{x} \times d t)$. Using continuity, $J(\mathbf{x}, t)=(1+o(1)) J(\mathbf{x}, 0)$ whence the integral is $(t+o(t)) \int_{B} J(\mathbf{x}, 0) d \mathbf{x}$ which matches the right hand side of (5.4).

Continuing the proof of Lemma 5.2, we observe that it suffices to show (5.3) for rectangles $K=$ $B \times T$. Let $B$ be a small ball about a point $\mathbf{y}$ and $T$ be a small rectangle $\left\{c_{j} \leq t_{j} \leq c_{j}+\Delta: 1 \leq j \leq k\right\}$ about a point $\mathbf{t}$. This makes $F \cdot \mathbf{n}$ roughly constant over the backward evolution of $B$ jumping at times in $T$, at any fixed time $s \leq t$, as long as the embedding sequence $\sigma$ is held fixed. Letting $t_{j+1}=t$ and $t_{0}=0$, we have

$$
|\eta(B, T, \sigma)| \sim|B||T| \exp \left[\sum_{j=0}^{k}-2(n-j)\left(t_{j+1}-t_{j}\right)\right] \prod_{j=1}^{k} \lambda_{j} .
$$

Here, $|\cdot|$ denotes Lebesgue measure, $F$ is the vector field $F(\mathbf{x})=A_{n-j+1} \mathbf{x}$, and $\lambda_{j}$ is $F \cdot \mathbf{n}$ evaluated at the point where the transition is made from dimension $n-j+1$ to dimension $n-j$. The asymptotic equivalence is as the diameter of $B$ goes to zero. The exponential reflects the fact that the backwards flow shrinks volume at a rate of $-2(n-j)$ over the time interval between $t_{j}$ and $t_{j+1}$.

The last step is to sum over $\sigma$. We do this inductively, beginning with the sum over all values of $\sigma_{1}$. Still supposing that $B$ is a small ball about $\mathbf{y}$ and $T$ is a small rectangle about $\mathbf{t}$, we see that as time goes backward from $t$ all the way to $t_{1}$, the backward evolution takes $\mathbf{y}$ to a point $\mathbf{y}^{\prime}=\left(y_{1}^{\prime}, \ldots y_{n-1}^{\prime}\right) \in \triangle_{n-1}$. The point $\mathbf{y}^{\prime}$ corresponds to one of $n+1$ possible points under the different possible choices of $\sigma_{1}=0, \ldots, n$, the $j^{\text {th }}$ of which is the point $\left(y_{1}^{\prime}, \ldots, y_{j}^{\prime}, y_{j}^{\prime}, \ldots, y_{n-1}^{\prime}\right)$ (where $j=0$ or $n$ corresponds to prepending 0 or appending 1). The identification $\sigma_{j}$ embeds $\triangle_{n-1}$ in $\triangle_{n}$ as the set of points whose $j$ and $j+1$ coordinates are equal. The dual vector $\mathbf{v} \mapsto \mathbf{v} \cdot \mathbf{n}$ is the functional $\mathbf{v} \mapsto v_{j+1}-v_{j}$. Therefore, at the point $\left(y_{1}^{\prime}, \ldots, y_{j}^{\prime}, y_{j}^{\prime}, \ldots, y_{n-1}^{\prime}\right)$, the value $\lambda_{j}=-F \cdot \mathbf{n}$ is $\left(y_{j+1}^{\prime}-2 y_{j}^{\prime}+y_{j}^{\prime}\right)-\left(y_{j}^{\prime}-2 y_{j}^{\prime}+y_{j-1}^{\prime}\right)$ which simplifies to $y_{j+1}^{\prime}-y_{j-1}^{\prime}$. Note that we have used $-F$ because Lemma 5.3 refers to the velocity of the backward flow. To summarize,

The factor $\lambda_{j}$ is the sum of the two gaps on either side of the coalesced point.

When $j=0$ or $n$ we obtain just one gap. Summing over values of $\sigma_{1}$ from 0 to $n$ gives twice the sum of all gaps, which is just the constant 2 .

Inductively, still restricting $B$ to a small ball about $\mathbf{y}$ and $T$ to a small rectangle about $\mathbf{t}$, we see that the sum over $\sigma_{1}, \ldots, \sigma_{i}$ of $\prod_{j=1}^{i} \lambda_{j}$ is $2^{i}$. When $i$ reaches $k$, we see that the sum over all $\sigma$ of $\prod_{j=1}^{k} \lambda_{j}$ is $2^{k}$. We have shown that summing over $\sigma$ leads to a constant factor. Letting $\Sigma$ denote 
the set of all embedding sequences, we see that that $\eta(\mathbf{y}, \mathbf{t}, \Sigma):=\sum_{\sigma \in \Sigma} \eta(\mathbf{y}, \mathbf{t}, \sigma)$ has density $2^{k}$ independent of $\mathbf{y}$. Integrating over $\mathbf{y}$ is therefore trivial and leads, for $T$ a small rectangle, to

$$
|\eta(B, T, \Sigma)|=|T| \cdot|B| \cdot 2^{k} \exp \left[-\sum_{j=0}^{k} 2(n-j)\left(t_{j+1}-t_{j}\right)\right] .
$$

Recalling that $\mu_{j}$ is $j$ ! times Lebesgue measure, we may rewrite this as

$$
\frac{1}{n !} \mu_{n}(\eta(B, T, \Sigma))=|T| \frac{\mu_{m}(B)}{m !} 2^{k} \exp \left[-\sum_{j=0}^{k} 2(n-j)\left(t_{j+1}-t_{j}\right)\right]
$$

hence

$$
\mu_{n}(\eta(B, T, \Sigma))=2^{k} \frac{n !}{m !}|T| \mu_{m}(B) \exp \left[-\sum_{j=0}^{k} 2(n-j)\left(t_{j+1}-t_{j}\right)\right] .
$$

Comparing to (5.2) with $k=n-m$ shows that for small rectangles $T$,

$$
\mu_{n}(\eta(B, T, \Sigma))=g(\mathbf{t})|T| \mu_{m}(B) .
$$

Integrating over $T$ yields the result for general sets $T$, which is (5.3), implying Lemma 5.2 and Theorem 2.1.

\section{The time Reversed Markov Chain on finite configurations}

The evolution of a finite configuration is deterministic. Nevertheless, for some choices of initial measure, projecting the configuration to its cardinality produces a continuous time Markov chain. The next result is an immediate consequence of Corollary 2.2. Recall that $\mu_{n}$ is the uniform measure on $\Delta_{n}$ and let $\mathcal{M}_{\lambda}$ denote a mixture of laws $\mu_{n}$ when $n$ has Poisson distribution with mean $\lambda$.

Proposition 6.1. For $\mathbf{x} \in \Delta$, let $N(\mathbf{x}, t)$ denote the number of particles in the configuration at time $t$ started from $\mathbf{x}$.

(i) If $\mathbf{x}$ is a random variable with law $\mu_{n}$, then $N(\mathbf{x}, \cdot)$ is a pure death Markov chain on $\mathbb{Z}^{+}$with initial law $\delta_{n}$ and jump rate at time $t$ from state $n$ given by $R(n, t)=2 n$ (hence transition kernel given by (5.1)).

(ii) If $\mathbf{x}$ is a Poisson with law $\mathcal{M}_{\lambda}$, then $N(\mathbf{x}, \cdot)$ is a pure death Markov chain on $\mathbb{Z}^{+}$whose initial distribution is Poisson $(\lambda)$ and whose transition rate is again $2 n$.

We now describe the time reversals of these two chains. Both time reversals are pure birth chains. The first chain is a time homogeneous chain. It starts at 1 and has rate $q(k, t):=2 k$ of jumping from $k$ to $k_{1}$. It is killed at the time $\sigma$ that it first reaches $n+1$. The second has jump rates that depend on time but not on state. The initial distribution is Poisson with a specified mean $\beta>0$. The rate of an upward jump at time $s$ is $r(k, s):=2 \beta e^{2 s} d s$. 


\section{Proposition 6.2.}

(i) Let $\nu_{n}$ be the law on right-continuous trajectories of the pure death Markov chain with death rate $2 k$ from state $k$, started from $\delta_{n}$ and run until the time $\tau$ that it reaches zero. For a trajectory $\omega$, denote the time reversal by $s \mapsto \omega^{\prime}(s):=\omega(\tau-s)$, made right continuous by setting $\omega^{\prime}(s)=\omega^{\prime}\left(s^{+}\right)$when $\tau-s$ is a jump time of $\omega$. Then the $\nu_{n}$ law of $\omega^{\prime}$ is the same as the law of a pure birth Markov chain with birth rate $2 k$ from state $k$ and initial distribution $\delta_{1}$, on the time interval $[0, \sigma)$, where $\sigma$ is the hitting time on $n+1$.

(ii) Fix $t$ and $\lambda>0$ and let $Q_{\lambda}$ be the law on right-continuous trajectories of the pure death Markov chain with death rate $2 k$ from state $k$, started from a Poisson of mean $\lambda$ and run for time precisely $t$. Let $\omega^{\prime}$ again denote the right-continuous reversal of the trajectory $\omega$, that is, $\omega^{\prime}(s)=\omega(t-s)$ except at jump times. Then the $Q_{\lambda}$ law of $\omega^{\prime}$ is the same as the law of a pure birth Markov chain with initial distribution Poisson of mean $\beta:=\lambda e^{-2 t}$ and birth rate $2 \beta e^{2 \lambda s}$ at time $s$, run for time precisely $t$.

Proof: Part (i): A trajectory $\omega$ is specified by its $n$ jump times, $s_{1}, s_{2}, \ldots, s_{n-1}, \tau$. The density of the trajectory with respect to $n$-dimensional Lebesgue measure is

$$
2^{n} n ! \exp \left(-2 n s_{1}-2(n-1)\left(s_{2}-s_{1}\right)-\cdots-2\left(\tau-s_{n}\right)\right) .
$$

Similarly, parametrizing trajectories of the birth chain by the jump times $\left(r_{1}, \ldots r_{n-1}, \sigma\right)$ gives a density of

$$
2^{n} n ! \exp \left(-2 r_{1}-4 r_{2}-\cdots-2 n\left(\sigma-r_{n-1}\right)\right) .
$$

Setting $\sigma=\tau$ and $r_{j}=\tau-s_{n-j}$ for $1 \leq j \leq n-1$ reduces both exponents to $-2 \tau-2 \sum_{j=1}^{n-1} s_{j}$.

For part (ii), begin by observing that a death rate of $2 n$ is equivalent to the $n$ particles each dying independently at rate 2 . Thus $Q_{\lambda}$ is the law of Poisson- $\lambda$ many particles each with an independent death time whose law has density $2 e^{-2 s}$ with an atom of size $e^{-2 t}$ being still alive at time $t$. A trajectory may be specified by death times together with the number remaining alive at time $t$, which is a Poisson process with intensity $2 \lambda e^{-2 s} d s+\lambda e^{-2 t} \delta_{t}$. The birth chain is specified by these same parameters. Clearly the number alive initially is Poisson with the correct mean $\beta=\lambda e^{-2 t}$. The arrival process has rate $2 \beta e^{2 \lambda s}=2 \lambda e^{-2 \lambda(t-s)}$ which agrees with the intensity of the point process of death times at time $t-s$. A Poisson process is completely specified by its intensity, establishing the distributional identity.

Let $R(n, t)$ be a jump rate for a pure birth process. We define an associated Markov transition kernel with state space $\Delta$ as follows. First a sample path of the birth process is generated, giving the jump times of the trajectory. Between jumps, the trajectory evolves deterministically via $\mathbf{x}^{\prime}=-A_{n} \mathbf{x}$ when $\mathbf{x} \in \Delta_{n}^{o}$. At jump times, a uniform $[0,1]$ random variable $U$ is generated, and the particle closest to $U$ splits (including the frozen particles at 0 and 1). Let $\mathbb{P}=\mathbb{P}_{\mu, \tau, R}$ denote the law of a trajectory with jump times given by $R$, started from $\mu$, with each trajectory $\omega^{\prime}$ stopped at time $\tau\left(\omega^{\prime}\right)$. 
Theorem 6.3 (time reversal).

(i) Let $R_{1}(k, t):=2 k$. Define $\mu *$ to be the measure giving probability $1 / 2$ to $\iota_{0,0}(0)$ and $1 / 2$ to $\iota_{0,1}(0)$. In other words, one of the frozen particles in the empty configuration is chosen by fair coin-flip to duplicate. Let $\tau$ be the hitting time on $\Delta_{n+1}$. Then the time reversal of $\mathbb{P}_{\mu *, \tau, R_{1}}$ is the law of forward evolutions started from $\mu_{n}$ and stopped upon hitting $\Delta_{0}$.

(ii) Let $R_{2}(k, t):=2 \beta e^{2 s}$. Let $\tau$ be a fixed constant, $t$. Then the time reversal of $\mathbb{P}_{\mathcal{M}_{\beta}, \tau, R_{2}}$ is the law of forward evolutions started from $\mathcal{M}_{\lambda}$ and stopped at time $t$.

Proof: Let $\Omega^{\prime}$ denote the space of reversals of finite trajectories of forward evolutions. The time reversal of the forward evolution from $\mu_{n}$ stopped at $\tau$ (respectively the forward evolution from $\mathcal{M}_{\lambda}$ stopped at $t$ ) is a well defined measure on $M_{1}$ (respectively $M_{2}$ ) on $\Omega^{\prime}$. By Proposition 6.2, the splitting times for $M_{1}$ (respectively $M_{2}$ ) are given by the birth process $R_{1}$ (respectively $R_{2}$ ). The only thing left to check is the law of the jump at the splitting times.

By (5.5), the jumps are chosen independently and proportionally to $\lambda_{j}$, the normal component of $F$ upon embedding by $\iota_{j}$. By (5.6), these are proportional to the sums of consecutive pairs of gaps. If $0=x_{0} \leq x_{1} \leq \cdots x_{k} \leq x_{k+1}=1$ and $U$ is a uniform random variable on $[0,1]$ then the index of $j$ such that $x_{j}$ is the nearest point to $U$ has law proportional to the sums of pairs of consecutive gaps. This completes the proof of the theorem.

\section{THE INFINITE SYSTEM AND A WEAK SOLUTION}

In this section we construct the infinite process, which is a probability measure $\mathbb{P}$ on the space of trajectories of infinite point configurations on $\mathbb{R}$. This requires constructing the space $\mathcal{M}$ of infinite point configurations, then the space of trajectories $\Omega$, then giving the probability measure $\mathbb{P}$. Although the usual choice for the space of infinite point configurations is that of counting measures, we use here a more direct construction that lends itself to taking limits of the finite configurations we have already defined.

Topologize $\Delta$ by treating the projection $\pi$ as describing attachment maps from $\partial \Delta_{n}$ to $\bigcup_{k=0}^{n-1} \Delta_{k}$ for each $n$. Notice that this identifies all $n$ faces of each $\partial \Delta_{n}$. Also note that $\Delta$ is not compact: a sequence of points in $\Delta_{n}^{o}$ for $n \rightarrow \infty$ has no limit. In this topology the trajectories $\left\{\Phi_{t}(\mathbf{x})\right\}$ are continuous. They are differentiable except at collision times; one-sided derivatives exist even at collision times.

Let $\Delta_{n}^{[a, b]}$ denote the simplex of vectors of length $n$ of elements of $[a, b]$ whose coordinates are nondecreasing, in other words, $\Delta_{n}$ with $[0,1]$ replaced by $[a, b]$; denote $\Delta_{n}^{(L)}:=\Delta_{n}^{[-L, L]}$. Let $\Delta^{[a, b]}$ denote the disjoint union of the interiors, also topologized by attachment. Each $\Delta^{[a, b]}$ embeds naturally in $\Delta^{[c, d]}$ for $[a, b] \subseteq[c, d]$. There are natural projections $\pi_{[c, d],[a, b]}: \Delta^{[c, d]} \rightarrow \Delta^{[a, b]}$ that ignore points outside of $[a, b]$ and act as one-sided inverses to the natural embeddings. We denote by 
$\pi_{[a, b]}$ the projection from the inverse limit to $\Delta^{[a, b]}$. Because $[a, b]$ may now vary, we will sometimes need the notation $\Phi_{t,[a, b]}(\mathbf{x})$ instead of $\Phi_{t}(\mathbf{x})$ for the time $t$ map on $\Delta^{[a, b]}$.

Let $\mathcal{M}$ denote the inverse limit of these projections as $M \rightarrow \infty$. Thus, for us, an infinite point configuration $\eta$ is a compatible collection of its finite projections rather than a counting measure. The projection $\pi_{[a, b]}$ induces a projection from $\mathcal{M}$ to trajectories on $\Delta^{[a, b]}$ via $\left(\pi_{[a, b]} \omega\right)(t):=\pi_{[a, b]}(\omega(t))$. Particles in the configuration $\pi_{[a, b]} \omega(t)$ die as they pass out of $[a, b]$ and are born as they pass into $[a, b]$; this does not create a discontinuity in $\pi_{[a, b]} \omega$ because of the identifications $\iota_{n, 0}$ and $\iota_{n, n+1}$ which allow creation and destruction at the endpoints of $[a, b]$. In this context, the Poisson measure with intensity $\lambda$, denoted $\mathcal{P}(\lambda)$, is the probability measure on $\mathcal{M}$ that projects under each $\pi_{[a, b]}$ to $\mathcal{P}(\lambda,[a, b])$.

Let $\Omega$ denote the space of continuous trajectories on $\mathcal{M}$, that is, continuous maps $\omega: \mathbb{R}^{+} \rightarrow \mathcal{M}$. Topologize $\Omega$ by uniform convergence on compact time intervals $[0, T]$. Here, by the inverse limit construction, uniform convergence means uniform convergence of each projection $\pi_{L} \omega(\cdot)$ on each compact time interval $[0, T]$. We care only about a tiny subset of $\Omega$, namely those trajectories that might arise as limits of trajectories $\Phi_{t, L}(\mathbf{x})$. To avoid having to craft arguments for paths that might have exotic behaviors, we define this closure, $\Omega_{0}$. Formally, for each $n$ and $L$ and each point $\mathbf{x} \in \Delta_{n}^{(L)}$, the trajectory $\left\{\Phi_{t, L}(\mathbf{x})\right\}$ lifts to an element of $\Omega$, the one in $\pi_{L}^{-1}$ with no points outside of $[-L, L]$. Let $\Omega^{o}$ denote the set of such trajectories and let $\Omega_{0}$ denote the closure of $\Omega^{o}$ in $\Omega$.

Our first aim is to show that elements of $\Omega_{0}$ look like trajectories of $\Phi$ : they can never uncoalesce and they satisfy the evolution rule (1.1). There is no probability involed here. A further goal will be to see that with probability 1 there are no multiple collisions and that in the limit the number of particles remains locally bounded in probability. We begin with formal definitions of collisions, of what it means to solve (1.1), and of how we count the number of particles in a spacetime rectangle.

Definition 7.1 (collision times). A collision time for a trajectory $\omega \in \Omega$ is a time $t$ such that for some $L, \varepsilon, n$ and $1 \leq j \leq n-1$, the trajectory $\pi_{L} \omega$ is in the interior of $\Delta_{n}^{(L)}$ for the time interval $(t-\varepsilon, t)$ and is in $\iota_{n-1, j} \Delta_{n-1}^{(L)}$ for the time interval $[t, t+\varepsilon)$. The location of the collision at time $t$ is the $j^{\text {th }}$ coordinate of $\omega(t)$. Although this will turn out to have probability zero, the definition of collision allows for more than one collision at time $t$ or for a multiple particle collision.

Definition 7.2 (solution).

(i) A trajectory $\omega \in \Omega$ is said to obey (1.1) if each $\pi_{L} \omega \in \Delta_{n}^{o}$ obeys (1.1) at time $t$ as long as $2 \leq i \leq n-1$ and $t$ is not a coalescing time for $x_{i}$.

(ii) A weak solution with Poisson- $\lambda$ initial conditions is a probability measure $\mathbb{P}_{\lambda}$ on $\Omega$ giving probability one to the set of trajectories obeying (1.1) and such that the law $\omega(0)$ under $\mathbb{P}_{\lambda}$ is Poisson with mean $\lambda$.

(iii) A strong solution with Poisson initial conditions is a map $\Xi$ from $\mathcal{M}$ to $\Omega$ such that $\Xi(\eta)(0)=\eta$ and $\Xi(\eta)$ obeys (1.1) for $\mathcal{P}(\lambda)$-almost every $\eta$. 
Definition 7.3 (occupation). Let $n(a, t)(\omega)=1$ if $\omega(t)$ has some coordinate equal to a and zero otherwise. Here the trajectory $\omega$ may have values in $\mathcal{M}$ or in any $\Delta^{[a, b]}$, noting that $n(c, t)\left(\pi_{[a, b]} \circ\right.$ $\omega)=n(c, t)(\omega)$ when $c \in[a, b]$. Let $n(A, t):=\sum_{x \in A} n(x, t)$ denote the occupation of the set $A$ by $\omega(t)$. Note that for $A=(-L, L)$ the value of $n(A, t)$ is the dimension $n$ of the face $\Delta_{n}^{(L)}$ such that $\pi_{L}(\omega(t)) \in \Delta_{n}^{(L)}$.

Most of the remainder of this section is devoted to proving Theorem 2.3. This will be proved by taking a weak limit of solutions to the finite system.

Proposition 7.4. Paths in $\Omega_{0}$ have the following properties.

(i) Paths never un-coalesce. Specifically, fix real $a<b$ and $0 \leq s<t$ and fix $\omega \in \Omega_{0}$. If $n(\{a, b\}, u)=0$ for all $s \leq u \leq t$, that is, no particle enters or exits $[a, b]$ during the time interval $[s, t]$, then $n([a, b], t)(\omega) \leq n([a, b], s)(\omega)$.

(ii) Paths in $\Omega_{0}$ satisfy (1.1).

Proof: For $(i)$, fix $\omega \in \Omega_{0}$. Choose a sequence $\left\{\omega_{L}: L \in \mathbb{Z}^{+}\right\}$converging to $\omega$ such that $\omega_{L}$ is a trajectory $\left\{\Phi_{t, L}\left(\mathbf{x}_{L}\right)\right\}$ for some point $\mathbf{x}_{L} \in \Delta^{(L)}$. Fix $s<t$ and an interval $[a, b]$ with $n(\{a, b\}, u)(\omega)=0$ for all $u \in[s, t]$. This implies $n(\{a, b\}, u)\left(\omega_{L}\right)=0$ for $L$ sufficiently large. Denote the initial number of particles in $[a, b]$ by $r:=n([a, b], s)(\omega)$. We need to show that $n([a, b], t) \leq r$.

Claim: If $\mathbf{x} \in \Delta^{(L)}$ has coordinates $x_{i}=y<z=x_{j}$ then the corresponding coordinates of $\Phi_{t, L}(\mathbf{x})$ differ by at most $(z-y) e^{2 t}$. Proof: Up to the first collision time, each gap $y_{k}:=x_{k}-x_{k-1}$ increases at rate at most $2 y_{k}$ due to the velocity of $x_{k}$ being at most $y_{k}$ and the velocity of $x_{k-1}$ being at least $-y_{k}$. This proves the claim up to the first collision time. Induction then proves it for all times.

Define the $\varepsilon$-mesh occupation of $[a, b]$ of a point $\mathbf{x} \in \Delta^{[a, b]}$, denoted $n_{\varepsilon}(\mathbf{x})$, to be the least $j$ such that the set of coordinates of $\mathbf{x}$ can be covered by $j$ intervals of length no more than $\varepsilon$. The fact that $\omega_{L} \rightarrow \omega$ implies that for every $\varepsilon>0$ there exists $M_{0}(\varepsilon)$ such that

$$
m \geq M_{0} \Rightarrow n_{\varepsilon}\left(\omega_{L}(s)\right)=r .
$$

Because the trajectories $\omega_{L}$ do not cross the endpoints $\{a, b\}$ in times in $[s, t]$, each particle at time $t$ comes from one or more coalesced particles at time $s$, hence from the claim we deduce that

$$
n_{e^{2(t-s)} \varepsilon}\left(\Phi_{t, L}\left(\mathbf{x}_{L}\right)\right) \leq n_{\varepsilon}\left(\mathbf{x}_{L}\right)=r .
$$

Sending $L \rightarrow \infty$ and noting that $\pi_{[a, b]} \omega$ is the uniform limit of $\pi_{a, b]} \omega_{L}$ on $[s, t]$, we see that $n_{\delta}(\omega(t)) \leq$ $r$ for any $\delta>e^{2(t-s)} \varepsilon$. Because $\varepsilon$ is arbitrary, this implies that $n([a, b], t) \leq r$.

For $(i i)$, let $x_{i}$ denote a particle for which $t$ is not a coalescing time. Uniform convergence of the $i-1, i$ and $i+1$ components of $\Phi_{t, L}\left(\mathbf{x}_{L}\right)$ implies that both the position and derivative of the particle $x_{i}$ converge uniformly. The time derivative in the limiting trajectory is therefore the limit of the derivatives for the finite trajectories, hence the limit trajectory obeys (1.1) at time $t$. 


\section{Lemma 7.5.}

(i) Let $\mathbf{x}^{(L)}$ have distribution $\mathcal{P}(\lambda, L)$. Any weak limit as $L \rightarrow \infty$ of the laws of the trajectories $\left\{\Phi_{t}\left(\mathbf{x}^{(L)}\right\}\right.$ is a weak solution with Poisson- $\lambda$ initial conditions. Consequently if the family of pushforwards of $\mathcal{P}(\lambda, L)$ under $\mathbf{x} \mapsto\left\{\Phi_{t, L}(\mathbf{x})\right\}$ is tight, then such a weak solution exists.

(ii) If for every $t$ the limit $\lim _{L \rightarrow \infty} \Phi_{t}\left(\pi_{L}(\eta)\right)$ exists $\mathcal{P}(\lambda)$-almost surely, then this limit defines a strong solution with Poisson- $\lambda$ initial conditions.

Proof: The weak limit of measures supported on a set $S$ is supported on the closure of $S$. Therefore, any weak limit of the trajectories $\Phi_{t, L}$ is in $\Omega_{0}$ and hence, by part (iii) of Proposition 7.4, obeys (1.1). The initial conditions are a weak limit of measures $\mathcal{P}(\lambda, L)$ which is the law $\mathcal{P}(\lambda)$ on $\Omega$. This is all that is needed for $(i)$ along with the observation that tightness of this family of laws implies the existence of a limit point. Statement (ii) is an immediate consequence of part (iii) of Proposition 7.4 and the definition of a strong solution.

All the time $t$ marginals of the finite system are explicitly known. The only work in establishing tightness is to check that the supremum over a time interval $s \in[0, t]$ of the number of particles in a fixed interval $[a, b]$ at time $s$ does not have positive mass going to infinity as $L \rightarrow \infty$. We have very little information about joint distributions of the process at two or more times. However, we can accomplish what we need by an identity that bounds the total occupation of the interval in terms of particle velocities.

Proposition 7.6. Let $N(a, t)(\omega):=\sum_{s \in[0, t]} n(a, s)(\omega)$ denote the cumulative occupation of $\{a\}$ up to time $t$. Then for any $n$, any time $t$ and any initial configuration $\mathbf{x} \in \Delta_{n}^{(L)}$,

$$
\int_{-L}^{L} N(x, t) d x \leq 2 L t
$$

Proof: Fixing $\mathbf{x}$, the successive collision times $\tau_{1}(\mathbf{x}), \tau_{2}(\mathbf{x}), \ldots$ may be treated as constants. Analyzing the trajectories separately on $\left[0, \tau_{1}\right),\left[\tau_{1}, \tau_{2}\right)$, etc., and summing the results, we may assume without loss of generality that $t<\tau_{1}$. The function $I(a, t)$ is the sum of $n$ indicators of graphs of functions, these functions being the trajectories of $x_{1}, \ldots, x_{n}$ as a function of time. Let $v_{j}(t)=2 x_{j}(t)-x_{j+1}(t)-x_{j-1}(t)$ denote the velocity of the $j^{t h}$ particle at time $t$, where we have used $x_{j}(t)$ to denote the $j^{t h}$ coordinate $\left(\Phi_{t}(\mathbf{x})\right)_{j}$ of the system at time $t$. One has an easy bound

$$
\sum_{j=1}^{n}\left|v_{j}(t)\right| \leq \sum_{j=1}^{n}\left(x_{j+1}-x_{j}\right)+\sum_{j=1}^{n}\left|x_{j}-x_{j+1}\right| \leq 2 L .
$$

Let $N(a, t, j)=\#\left\{s \in[0, t]: x_{j}(t)=1\right\}$ count incidences for just the $j^{t h}$ particle. Banach's Indicatrix Theorem [Ban25, Théorèmes 1 and 2] (see also [Nat55]) states that $\int_{-L}^{L} N(x, t, j) d x$ is equal to the total variation of the trajectory $\left\{x_{j}(s): 0 \leq s \leq t\right\}$. This is just $\int_{0}^{t}\left|v_{j}(s)\right| d s$. Summing gives

$$
\int_{-L}^{L} N(x, t)=\sum_{j=1}^{n} \int_{-L}^{L} N(x, t, j) d x=\sum_{j=1}^{n} \int_{0}^{t}\left|v_{j}(s)\right| d s .
$$


Everything is nonnegative and we may interchange the integral and sum to obtain

$$
\int_{-L}^{L} N(x, t) d x=\int_{0}^{t}\left(\sum_{j=1}^{n}\left|v_{j}(s)\right|\right) d s \leq \int_{0}^{t} 2 L d s=2 L t .
$$

Lemma 7.7. For any $n$, any time $t$, any $L$, and any interval $[a, b] \subseteq[-L, L]$,

$$
\mathbb{E} \int_{a}^{b} N(x, t) d x \leq 2(b-a) t+2 t \lambda^{-1} e^{2 t},
$$

where the expectation is with respect to trajectories whose intial point is uniform on $\Delta_{n}^{(L)}$.

Proof: As in the previous proposition,

$$
\int_{a}^{b} N(x, t) d x=\int_{0}^{t} V(s) d s
$$

where $V(s)=\sum_{j}\left|v_{j}(s)\right|$ is the sum of the speeds of all particles in $[a, b]$ at time $s$. Instead of $V(s) \leq 2 L$ for all $s$, we have

$$
V(s) \leq 2(b-a)+(M(s)-b)+(a-m(s))
$$

where $M(s)$ is the location of the first particle to the right of $b$ at time $s$ and $m(s)$ is the location of the first particle to the left of $a$ at time $s$. Each of the terms $M(s)-b$ and $a-m(s)$ is an exponential of mean $\lambda^{-1} e^{2 s}$, truncated to $L-b$ and $a+L$ respectively. The mean of the truncated exponential is at most the mean of the untruncated exponential, leading to

$$
V(s) \leq 2(b-a)+2 \lambda^{-1} e^{2 s} .
$$

Plugging this into (7.2) proves the lemma.

Lemma 7.8 (velocities are bounded in probability). Fix $[a, b]$ and $T$. For any trajectory $\omega:=$ $\left\{\Phi_{t, L}(\mathbf{x})\right\}$ in $\Omega^{o}$, let $V(\omega)$ denote the maximum absolute velocity of any particle in $[a, b]$ at any time $t \leq T$. Then under Poisson initial conditions, $V$ is bounded in probability, meaning that

$$
\mathcal{P}(\lambda, L)(\{\omega: V(\omega) \geq M\}) \leq g(M)
$$

for some function $g$ going to zero as $M \rightarrow \infty$ and depending on $a, b$ and $T$ but not $L$.

Proof: Label each initial particle $x_{j}$ by the positive real number $\ell\left(x_{j}\right):=e^{2 T} \max \left\{x_{j}-x_{j-1}, x_{j+1}-\right.$ $\left.x_{j}\right\}$. From the claim proved in Proposition 7.4 we know that at all times between 0 and $T$, the gaps between $x_{j}$ and $x_{j-1}$ is at most $\ell\left(x_{j}\right)$ provided that we interpret the gap to be zero if the two particles have coalesced. The same is true of the gap between $x_{j}$ and $x_{j+1}$. It follows that the two gaps adjacent to a particle $x$ at any time up to $T$ is at most the maximum, call it $W(x)$, of $\ell\left(x_{j}\right)$ over all particles $x_{j}$ that have coalesced into $x$. Because the velocity of any particle at any time is bounded by the maximum adjacent gap, the position of any particle $x$ at time $t \leq T$ must be within distance $t W(x)$ of the initial position of some $x_{j}$ that has coalesced into $x$. If the particle $x$ at time 
$t \leq T$ is in the interval $[a, b]$, and if $x_{j}$ is the initial position of a particle that has coalesced into $x$, then the distance $d\left(x_{j},[a, b]\right)$ from $x_{j}$ to the interval $[a, b]$ must be at most $T W(x)$. Choosing $j$ for which $W(x)=\ell\left(x_{j}\right)$, we see that $d\left(x_{j},[a, b]\right) \leq T \ell\left(x_{j}\right)$. Therefore,

$$
V(\omega) \leq V_{*}(\omega):=T \cdot \sup \left\{\ell\left(x_{j}\right): d\left(x_{j},[a, b]\right) \leq T \ell\left(x_{j}\right)\right\} .
$$

Couple the distributions of $V_{*}$ under the laws $\mathcal{P}(\lambda, L)$ as $L$ varies, by taking the restrictions to $[-L, L]$ of a single pick $\eta$ from the Poisson measure $\mathcal{P}(\lambda)$ on $\mathbb{R}$. The function $\ell\left(x_{j}\right)$ for particles of $\pi_{L} \eta$ are at most what they are for the corresponding particles of $\eta$, with equality except for the first and last particles in $[-L, L]$. The conclusion of the lemma will therefore follows once we show that for infinite configurations,

$$
\mathcal{P}(\lambda)\left[\left\{\eta: V_{*}(\eta)=\infty\right\}\right]=0 .
$$

This follows from the fact that the supremum in (7.3) is taken over an almost surely finite set. This, in turn, is a simple consequence of the Borel-Cantelli lemma, once one observes that the probabilities $q_{n}$ and $q_{n}^{\prime}$ are summable, where $q_{n}$ is the probability of existence of an initial particle $x$ in $[b+n, b+n+1]$ with $T \ell(x) \geq n$ and $q_{n}^{\prime}$ is the analogous probability for a particle in $[a-n-1, a-n]$.

Proof of Theorem 2.3. Fix $L, \lambda>0$. On the probability space of trajectories with initial distribution $\mathcal{P}(\lambda, L)$, define random variables $Y=Y(L, a, b, t)$ to be the maximum number of particles in the interval $[a, b]$ at any time $s \in[0, t]$. Any particle contributing to this number is either in $[a-1, b+1]$ at time zero or crosses the interval $[a-1, a]$ or crosses the interval $[b, b+1]$. A particle crossing an interval $J$ of length 1 contributes at least 1 to $\int_{J} N(x, t) d x$. Therefore,

$$
\mathbb{P}(Y>3 y) \leq \mathbb{P}\left(Y_{0}>y\right)+\mathbb{P}\left(Y_{1}>y\right)+\mathbb{P}\left(Y_{2}>y\right)
$$

where $Y_{0}$ is the initial number of particles in $[a, b], Y_{1}=\int_{a-1}^{a} N(x, t) d t$ and $Y_{2}=\int_{b}^{b+1} N(x, t) d t$. Each of $Y_{0}, Y_{1}$ and $Y_{2}$ is bounded in expectation by a constant depending on $a, b, t$ and $\lambda$ but not $L$. Therefore, $\mathbb{P}(Y>3 y) \leq C / y$, finishing the proof of tightness.

Tightness of these variables for all $a, b$ is tightness of the pushforwards in part $(i)$ of Lemma 7.5, the lemma then implying existence of the weak solution which we denote henceforth $\left\{\Psi_{t}\right\}$. By construction the two-sided derivative of each particle's position exists away from its collision times and (1.1) is satisfied.

To argue for almost sure distinctness of collision times, fix $\lambda$ and $T$ and the interval $[a, b]$. For $\varepsilon>0$, define the event $G_{\varepsilon}$ of an $\varepsilon$-almost multiple collision by time $T$ on $[a, b]$ to be the event that for some times $s$ and $t \in[s, s+\varepsilon]$ there are distinct pairs of particles whose positions differ by at most $\varepsilon$ (the two pairs can share one particle but not both). If $\omega_{L} \in \Omega^{\circ}$ with $\omega_{L} \rightarrow \omega$, and $\omega$ has a multiple collision at some time $t \leq T$, then for all $\varepsilon>0$ there will be $L_{0}(\varepsilon)$ such that $L \geq L_{0}$ implies $\omega_{L}$ has an $\varepsilon$-almost multiple collision. Therefore, to finish the proof of the theorem, it suffices to show that the probability of $G_{\varepsilon}$ under any $\mathcal{P}(\lambda, L)$ is bounded above by some function $\kappa(\varepsilon)$ going to zero as $\varepsilon \rightarrow 0$, independent of $L$. 
Fix an integer $M>0$ and let $H_{M}$ denote the event that the maximum absolute velocity of any particle in $[a, b]$ up to time $T$ is at most $M$. Let $S$ be the set of multiples of $\varepsilon$ in $[0, T]$. Then, on $H_{M}$, the event of an $\varepsilon$-almost multiple collision implies that for some time $s \in S$, there are two distinct pairs of particles (possibly sharing one particle) within distance $(M+1) \varepsilon$ at time $s$. Any pair of points in $[a, b]$ within distance $(M+1) \varepsilon$ of each other is in the same interval $[x, x+2(M+1) \varepsilon]$ for some $x=k(M+1) \varepsilon, 0 \leq k \leq 2(b-a) /((M+1) \varepsilon)$. Therefore, on $H_{M}$, the event of an $\varepsilon$-almost collision is contained in the union of at most $|S|\left(2(b-a) \varepsilon^{-1} /(M+1)\right)^{2}$ events that two distinct pairs of points at a specific time $s$ are in two distinct intervals $\left[x_{1}, x_{1}+2(M+1) \varepsilon\right]$ and $\left[x_{2}, x_{2}+2(M+1) \varepsilon\right]$, together with the $|S| 2(b-a) \varepsilon^{-1} /(M+1)$ events that two distinct pairs of points at a specific time $s$ are together in the same interval $[x, x+2(M+1) \varepsilon]$.

We bound this from above using the fact that the time $s$ marginal of the number of points in any interval of length $c$ is a Poisson of mean $\lambda e^{-2 s} c \leq \lambda c$. The probability of a Poisson with mean $\nu$ being at least 2 is at most $\nu^{2} / 2$ and the probability of it being at least 3 is at most $\nu^{3} / 6$. Therefore, applying the bound with $c=2(M+1) \varepsilon$, and using the bound $|S| \leq T \varepsilon^{-1}+1$, we see that the probability of an $\varepsilon$-almost collision is at most

$$
\left(T \varepsilon^{-1}+1\right) \frac{2(b-a)}{M+1} \varepsilon^{-1} \frac{(2(M+1) \varepsilon)^{3}}{6}+\left(T \varepsilon^{-1}+1\right)\left(\frac{2(b-a)}{M+1} \varepsilon^{-1}\right)^{2}\left(\frac{(2(M+1) \varepsilon)^{2}}{2}\right)^{2} .
$$

Clearing away irrelevant stuff, we see that

$$
\mathcal{P}(\lambda, L)\left(H_{M} \cap G_{\varepsilon}\right) \leq C T(b-a)^{2} M^{2} \varepsilon .
$$

By Lemma 7.8 , the probability of $H_{M}^{c}$ is bounded above by $g(M)$. Choosing $M=\varepsilon^{-1 / 3}$ we see that

$$
\mathcal{P}(\lambda, L)\left(G_{\varepsilon}\right) \leq C T(b-a)^{2} \varepsilon^{1 / 3}+g\left(\varepsilon^{-1 / 3}\right) .
$$

This goes to zero as $\varepsilon \downarrow 0$, finishing the proof of Theorem 2.3.

\section{Further COMments And QUeStions}

The most glaring absence of a result concerns strong solutions. The following conjecture, together with Lemma 7.5 would imply the existence of a strong solution to (1.1). A proof seems not too far off via results along the lines of Lemma 7.7.

Conjecture 8.1 (strong solution). For $\mathbb{P}$-almost every $N \in \Omega$, the limit

$$
\Psi_{t}(N):=\lim _{L \rightarrow \infty} \Psi_{t}^{(L)}(N)
$$

exists and defines a trajectory $t \mapsto \Psi_{t}(N)$.

Conjecture 8.1 also implies the following restatement of Theorem 2.4 in terms of the evolution $\left\{\Psi_{t}\right\}$.

$$
\mathcal{P}(\lambda) \circ \Psi_{t}^{-1}=\mathcal{P}\left(\lambda e^{-2 t}\right) .
$$


Numerical simulation suggests a substantially more general result than those obtained in this paper. We believe that the Poisson distribution is an attracting fixed point for the dynamics: any reasonable initial measure, if allowed to evolve under these dynamics and rescaled, should converge Poisson. There is some numerical evidence for this. In one experiment, 250 million points were initially placed on the unit interval with uniform density and periodic boundary conditions. New points were then placed halfway between pairs of adjacent points, and the initial points were removed. The resulting configuration is known as the 1D Poisson-Voronoi configuration. Its cell sizes are onedependent but not independent, with marginal density $4 x \exp (-2 x)$. Figure 8 illustrates histograms of the normalized cell sizes as time grows. As increasingly many of the cells disappear, the pdf approaches the exponential distribution, the cell size marginal for the Poisson. The data is also compatible with asymptotic independence of neighboring cell sizes. Additional supporting data from this initial configuration and several other initial configurations are reported in [Laz11].

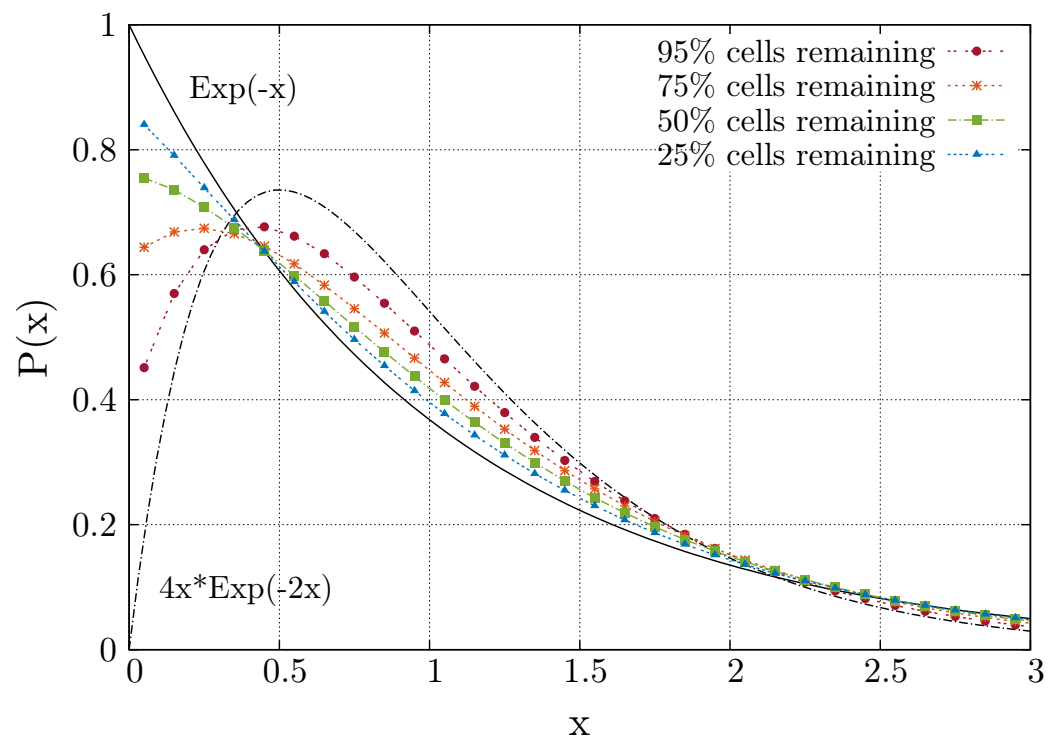

To make this into a precise conjecture, let $\nu$ be a probability measure on $\mathbb{R}$ with finite mean and let $Q_{\nu}$ be the stationary renewal process on $\mathbb{R}$ with renewal distribution $\nu$. Intuitively, $Q_{\nu}$ is the law of a random configuration of points whose gaps are IID $\nu$.

Conjecture 8.2. For any $\nu$ on $\mathbb{R}$ with mean $m<\infty$, there is a weak solution $\mathbb{P}_{\nu}$ to (1.1) with initial conditions $Q_{\nu}$. Let $\nu_{t}$ be the time $t$ law of such a weak solution, with space rescaled by $e^{-2 t}$. Then $\nu_{t} \rightarrow \mathcal{P}(1 / m)$ weakly as $t \rightarrow \infty$.

This conjecture is the concrete assertion of the universality mentioned in the abstract. While a proof seems farther off for this conjecture than for Conjecture 8.1] we hope to attack it via the 
dual process introduced in Proposition 6.2. While the forward evolution is deterministic, the timereversed process has randomness. We believe this will help us to show that running the dual process back in time $t$ units produces, as $t \rightarrow \infty$, a configuration asymptotically independent from any $Q_{\nu}$.

\section{ACKNOWLEDGEMENTS}

The first author would like to thank Benjamin Matschke and Amanda Redlich for helpful discussions. The second author would like to thank Ryan Hynd and Herman Gluck for helpful conversations. Thanks also to Moe Hirsch and Joel Robbin for providing references to the works of J. Aubin and P. Hartman.

\section{REFERENCES}

[Aub77] J. Aubin. Applied Abstract Analysis. John Wiley and Sons, New York, 1977.

[Ban25] S. Banach. Sure les lignes rectifiables et les surfaces dont l'aire est finie. Fund. Math., 7:225-236, 1925.

[CP92] Jack Carr and Robert Pego. Self-similarity in a coarsening model in one dimension. Proceedings of the Royal Society of London. Series A: Mathematical and Physical Sciences, 436(1898):569-583, 1992.

[Der95] B Derrida. Coarsening phenomena in one dimension. In Complex Systems and Binary Networks, pages 165-182. Springer, 1995.

[Der97] B Derrida. Non-trivial exponents in coarsening phenomena. Physica D: Nonlinear Phenomena, 103(1):466477, 1997.

[FRS92] MA Fortes, VA Ramos, and Amilcar Soares. Grain growth in one dimension: The mean field approach. In Materials Science Forum, volume 94, pages 337-344. Trans Tech Publ, 1992.

[Har72] P. Hartman. On invariant sets and on a theorem of waewski. Proc. Amer. Math. Soc., 32:511-520, 1972.

[HR96] O Hunderi and N Ryum. The influence of spatial grain size correlation on normal grain growth in one dimension. Acta materialia, 44(4):1673-1680, 1996.

[HRW79] O Hunderi, N Ryum, and H Westengen. Computer simulation of grain growth. Acta Metallurgica, 27(2):161-165, 1979.

[Laz11] Emanuel A Lazar. The evolution of cellular structures via curvature flow. PhD thesis, Princeton, 2011.

[Lee03] J. Lee. Introduction to Smooth Manifolds, volume 218 of Lecture Notes in Mathematics. Springer, New York, 2003.

[LMMS11] Emanuel A Lazar, Jeremy K Mason, Robert D MacPherson, and David J Srolovitz. A more accurate three-dimensional grain growth algorithm. Acta Materialia, 59(17):6837-6847, 2011.

[MLMS12] JK Mason, EA Lazar, RD MacPherson, and David J Srolovitz. Statistical topology of cellular networks in two and three dimensions. Physical Review E, 86(5):051128, 2012.

[Mul86] WW Mullins. The statistical self-similarity hypothesis in grain growth and particle coarsening. Journal of Applied Physics, 59(4):1341-1349, 1986.

[Mul91] WW Mullins. A one dimensional nearest neighbor model of coarsening. Technical report, Carnegie Mellon University, Department of Mathematical Sciences, http://repository.cmu.edu/math, 1991.

[MV89] WW Mullins and Jorge Viñals. Self-similarity and growth kinetics driven by surface free energy reduction. Acta metallurgica, 37(4):991-997, 1989.

[Nat55] I. P. Natanson. Theory of functions of a real variable. Frederick Ungar Publishing Company, New York, 1955. 
Laboratory for Research on the Structure of Matter, University of Pennsylvania, Philadelphia, PA 19104

E-mail address: mlazar@seas.upenn.edu

$U R L$ : www.seas .upenn.edu/ ${ }^{\sim m l a z a r /}$

Department of Mathematics, University of Pennsylvania, Philadelphia, PA 19104

E-mail address: pemantle@math.upenn.edu

$U R L:$ www.math.upenn.edu/ pemantle/ 